\title{
El Estado de Derecho brasileño y LA NECESIDAD DE RESPETO A LOS PRECEDENTES VINCULANTES
}

Paula Pessoa Pereira

\begin{abstract}
RESUMEN:
La lucha por el Estado de Derecho implica la limitación de la actividad estatal al derecho como resultado del cumplimiento del objetivo del sistema, con el fin de favorecer una situación más ventajosa para el individuo. Este trabajo estudiará la teoría de los precedentes vinculantes como medio para asegurar la protección de la seguridad jurídica, su previsibilidad y su certeza. Es cierto que la resolución de los precedentes judiciales vinculantes no agota los problemas de la relación entre el poder y la ley. Sin embargo, permitirá la clausura del sistema jurídico en lo que respecta a controlar las acciones del poder judicial que deben ser guiadas por la coherencia, la igualdad de las decisiones en casos similares y la seguridad jurídica.
\end{abstract}

Palabras clave: Estado de Derecho, jurisdicción contemporánea, seguridad jurídica, precedentes vinculantes.

\section{Abstract:}

The struggle for the rule of law implies the limitation of state activity to the law, as a result of compliance with the objective of the system, in order to favor a more advantageous to the individual. In order to solve this problem this paper aims to study the theory of binding precedents as a mean to ensure the

* Estudiante del Máster en Derecho Procesal Civil de la Universidad Federal del Paraná, Brasil. Especialista en Derecho Procesal Civil por la Universidad Federal de Bahia, Brasil. Analista Procesal en el Tribunal del Trabajo de la Novena Región (Paraná, Brasil). Dirección: Rua Dr. Pedrosa, n 152, apt ${ }^{\circ}$ 1904, Centro, Curitiba/PR, 80420-120. Teléfono: (55) 41 9995-1393. Dirección electrónica: pessoapp@hotmail.com.

El artículo es inédito. 
protection of legal certainty and predictability. It is true that the resolution of binding judicial precedent does not exhaust the problems of the relationship between power and law. However, the enclosure will allow the legal system in regarding to control of judicial acts, to be guided by consistency, equality of decisions in similar cases and legal certainty.

Key words: Rule of law, jurisdiction contemporary, legal certainty, binding precedents.

\section{INTRODUCCIÓN}

Una expresión que siempre ha fascinado a los abogados -y que aún sigue fascinando- es el Estado de Derecho. No obstante su comprensión simple y obvia, se trata de muchos problemas y una demanda excesiva, toda la actividad estatal está sujeta a los mandatos de la ley ${ }^{1}$. Por un lado, hay un límite legal del poder político en la cara del Estado de Derecho, y por el otro, una normatividad legal que cubre todas las necesidades de la sociedad dando forma a las relaciones sociales.

La demanda más básica de los ciudadanos frente al Estado de Derecho es que este contribuya a la paz social, lo cual proporciona condiciones para la civilidad y las relaciones jurídicas constituidas en la comunidad. Sin embargo, no se puede pensar que sea esta una tarea que el Estado puede ejercer indefinidamente, sin la observancia de un parámetro de control. Por el contrario, es en el derecho que el Estado encuentra el límite de su atuación, una relación crucial para la realización de las condiciones de justicia social y la civilidad.

Una definición pacífica de la fórmula Estado de Derecho casi no se puede obtener, ya sea por los diferentes conceptos desarrollados a partir de las tradiciones históricas diferentes, es asumido por los diversos significados en diversos contextos de la evolución histórica. Estos factores deben tenerse en cuenta cuando se trata de definir los límites y alcances de la norma de la ley.

Breve comparación entre las tradiciones jurídicas de la literatura occidental en los solicitantes históricos, a saber, alemán, francés y anglo-americanos (llamados, respectivamente, Rechtsstaat, État de Droit y Estado de Derecho), permiten una mejor comprensión de ciertos matices básicos del concepto de Estado de Derecho, así como el intercambio de elementos básicos en común, tales como la relación entre el Estado y la institucionalización de un marco, es decir, el deber del Estado para ejercer su poder a través de leyes, de acuerdo con los principios de legalidad ${ }^{2}$.

Por lo tanto, la definición esencial de los puntos cardinales que permiten la realización material del Estado de Derecho, a fin de comprender las mutaciones

1 VERDÚ, Pablo Lucas. A luta pelo Estado de Direito. Rio de Janeiro: Editora Forense, 2007. p. 2.

2 ROSENFELD, Michel. "The rule of law and the legitimacy of constitutional democracy". Southern California Law Review, vol. 74, 2001. pp. 1318-1337. 
conceptuales en los que esta fórmula probada, siempre con miras a su destino final, que es la ventaja de los individuos. Ellos son el poder político, la ley y los individuos ${ }^{3}$.

Sucede que, en nuestros tiempos, el Estado de Derecho, llamado Estado Constitucional, presenta numerosos problemas para su realización. Esto sucede, entre otras razones, porque la noción de integridad de la ley y la autosuficiencia de la Ley Orgánica hizo ruínas la visión actual de los requisitos de la ciencia jurídica y la nueva sociedad política organizada.

Entre los muchos factores que provocan el replanteamiento de la expresión "Estado de Derecho", este ensayo se centrará en el problema de la legislación incompleta y abierta, que su demanda por un papel predominante de los jueces en la definición del derecho -mostrando la vocación de nuestro tiempo a un tribunal estatal- implica una situación de crisis de la seguridad jurídica, lo que deviene en una desventaja para los ciudadanos.

Teniendo en cuenta factores tales como el propósito del Estado de Derecho para garantizar la protección social, la paz, la seguridad jurídica y la libertad, la vocación de nuestro tiempo hacia un Estado jurisdiccional, las fluctuaciones en la definición de los derechos de los tribunales, un sistema marcado por el valor de la independencia del juez, cabe pensar que - para ganar la lucha por el Estado de Derecho- será esencial el estudio y desarrollo de la teoría de los precedentes vinculantes.

De hecho, la adopción de la teoría del respeto a los precedentes judiciales aparece como un intento para contestar a la siguiente pregunta: ¿La respuesta del control del poder está en el derecho o en el exterior? ${ }^{4}$ El respeto por los precedentes permite el control de las garantias de los derechos a todos los ciudadanos por igual, así como la unidad y coherencia del ordenamiento jurídico en sí.

\section{El Estado de Derecho y SUS TiEMpos históricos}

La regla de la fórmula del Estado de Derecho, tan querido y familiar para los abogados, en el curso del tiempo histórico no ha mantenido una unidad conceptual, en cambio, ha desarrollado diferentes significados y asignaciones definitivas a cada contexto socio-histórico involucrado.

Sin embargo, no se quiere con esta afirmación conclur que el proceso histórico de formación y maduración de la definición del Estado de Derecho se ha producido

MARMOR, Andrei. "The Rule of Law and Its Limits" (April 2003). USC Law and Public Policy Research Paper No. 03-16. Disponible en: http://ssrn.com/abstract=424613.

4 Es necesario aclarar en este punto que el trabajo propuesto no es universal ni pretende responder a las preguntas del cierre completo del sistema legal, ni a la cuestión de quién controla el poder, sólo trata de presentar una solución a una de las facetas del problema destino final del Estado de Derecho. 
de forma lineal. Es cierto que la historia no se compone de eventos lineales, todo lo contrario, sus acontecimientos y movimientos se materializan de formas diversas y, en algunos casos, opuestas.

En este sentido se desarrolla la investigación del jurista italiano Pietro Costa en su ensayo El Estado de Derecho: Una introducción histórica. Aquí, el profesor florentino trata de los diversos significados y los problemas del Estado de Derecho en tiempos históricos diferentes, adoptando, como puntos cardinales de este, el poder político (soberanía y Estado), el derecho (con el propósito derecho y las normas) y los individuos. En pocas palabras, la idea que subyace a todo el texto es la cuestión de la relación entre el poder y el derecho (marcado por la tensión y la continuidad) en una comunidad jurídica particular y en un momento histórico determinado ${ }^{5}$.

Por lo tanto, se propone una división en tres períodos históricos en cuyo caso el Estado de Derecho se desarrolla. Éstas se presentan en orden de proximidad con el tema, a saber:

- La primera vez es la historia. Historia en el sentido estricto del Estado de Derecho que comienza desde el momento en que hay una expresión léxica en cuestión.

- La segunda es la pre-historia. Identificados en los contextos y tiempos en que se reconocen los rasgos de una posición en la cual el Estado de Derecho se encuentra su formulación explícita.

- La tercera vez. En este sentido, el tema del poder-deber es rica y plena, no obstante algunos puntos de vista culturalmente muy distantes de lo políticolegal que construyó la pre-historia de la regla del concepto de la ley.

Dentro de la idea esbozada por Pietro Costa, el horizonte de sentido de la regla de la ley surge en un escenario temporal muy amplio que incluye el mundo antiguo y la cultura medieval, en la medida en que pretende ser una expresión de confianza que restablece el derecho de los individuos, es decir, la norma objetiva, como una manera de detener o de regular la energía desenfrenada de la soberanía.

Dice el jurista florentino que uno no necesita esperar a la Edad Moderna para encontrar el tema de la dicotomía que opone un tipo de régimen al otro, tomando esto como criterio para distinguir entre el gobierno y la ley. Ejemplo de

$5 \quad$ El profesor explica: "é igualmente plausível atribuir ao problema do qual a fórmula 'Estado de Direito' quer ser uma solução, um cenário temporalmente bem mais amplo e em suma coincidente com a história político-intelectual do Ocidente, visto que nela surge sempre de novo a exigência de sublinhar a inevitável tensão (e a necessária conexão) entre poder e direito". COSTA, Pietro. "O estado de direito: uma introdução histórica". En: COSTA, Pietro; ZOLO, Danilo (Org.) O estado de direito, história, teoria, crítica. São Paulo: Martins Fontes, 2006. p. 98. 
lo anterior puede encontrarse en el mundo antiguo, tanto en Platón y Aristóteles, que, a pesar de la diversidad de sus filosofías políticas y jurídicas -metodológicas y sustantivas-, han discutido el problema de las formas de gobierno, poniendo de relieve el papel central de la ley. De hecho, la tensión entre su carácter general y manifestaciones siempre varios de la unicidad.

La difícil composición entre decisión despotica y el respeto a un orden normativo, son temas siempre ausentes presentes en la reflexión en el mundo antiguo y medieval heredado, en profundidad.

Para los teólogos medievales y los juristas, la imagen del poder es inseparable de la idea de un orden normativo en el que las voluntades individuales se organizan de acuerdo a las jerarquías naturales que consisten en las estructuras fundamentales del cosmos y la sociedad. El poder tiene su expresión en la jurisdicción en una ley que hable de llevar a cabo la esencia del poder, precisamente porque supone la facultad de ordenar y declarar.

En este punto, pertinente la aclaración de Pietro Costa sobre la existencia de un error contenido en la afirmación común de que el absolutismo de los siglos XVII y XVIII se caracterizó por un poder ilimitado y desenfrenado, el autor explica que el absolutista soberano, lejos de tener un poder ilimitado, posee un poder muy limitado, ya que él se vio obligado a tener en cuenta los marcos normativos, estructuras institucionales, los derechos y privilegios de los cuerpos y las ciudades, en gran medida independientes que lo condicionaban ${ }^{6}$.

En la edad moderna, más precisamente durante el siglo XIX, fue cuando, en términos generales, el Estado de Derecho encontró su horizonte de sentido en el nexo poder-derecho, por el intento de juridización del poder. Fue en este momento histórico que la expresión Estado de Derecho adquirió la convicción de que el derecho puede controlar de forma efectiva el poder, gracias a los servicios desarrollados por la ciencia moderna del derecho público.

Sin embargo, en el período posterior a la Segunda Guerra Mundial, muchos de los temas que hasta entonces parecían resueltos en torno a la fórmula del Estado de Derecho, volvieron a aparecer vestidos con nuevos problemas. La transformación de la función del derecho, la pérdida de su ilustración y su uso cada vez más como un instrumento de la acción del gobierno y no como una representación de la voluntad general del pueblo, el papel del juez y su relación com el control de las leyes y la observancia de un parámetro normativo, son cuestiones que reproponen la configuración y la definición del Estado de Derecho.

Op. cit. p. 102. 


\section{NeCESidAd PARA El deSARROLlo de UNA TEOría de los PRECEDENTES VINCULANTES}

El Estado de Derecho es la piedra angular del sistema constitucional contemporáneo, debido al desarrollo de su papel en la consolidación de las recientes transiciones de regímenes autoritarios o totalitarios para las democracias constitucionales en la Europa continental ${ }^{7}$. En términos más generales, el Estado de Derecho requiere que la función legislativa del Estado se separe de la función jurisdiccional y que nadie en la política esté por encima de la Constitución. Podemos resumir las características esenciales del Estado de Derecho como un Estado constitucional en: lo que limita los poderes del gobierno; lo que ofrece la protección de los derechos fundamentales; y en lo que realiza el control de constitucionalidad de las leyes.

De hecho, alrededor de la historia de la lucha por el Estado de Derecho, en lo que aquí nos ocupa, podemos decir que pasó por momentos vocacionales. Me explico: el siglo XIX se dedicó a la ley y la autonomía jurídica de la ciencia; el siglo XX por la codificación; por la decodificación, a finales de este siglo; y en el naciente siglo XXI por la jurisdicción y la doctrina jurídica ${ }^{8}$.

¿Y por qué la vocación del siglo por la jurisprudencia? Una respuesta a esta pregunta, adoptada aquí, es que, más importante que el ejercicio del poder y la forma de legitimación, es su control. En este paso, el rendimiento del control del poder (del Estado) requiere una adecuada organización de las instituciones y, por lo tanto, del desarrollo de la jurisdicción. De allí que, como el poder es limitado por el derecho, nada más consecuente que el agigantamento del desempeño de los jueces en la medida que la aplicación e interpretación de lo que constituye el derecho compete a ellos?.

$7 \quad$ Destacamos aquí la advertencia del profesor Pietro Costa: "La variedad de matices que caracterizan el Estado de Derecho en su parábola, no parece salir de una relación histórica entre la norma imperativa de derecho y el orden determinado desde lo político-constitucional. Sin duda, sí hay un nexo entre el Estado históricamente prevaleciente de la ley y el constitucionalismo liberal, la historia posterior, del siglo XIX, el imperio de la atención y la ley sobre los usos diversos de esta fórmula que ahora se refiere, de acuerdo con las circunstancias, el Estado fascista y también para el estado social de la época después de la Segunda Guerra Mundial". Op. cit. p. 194.

$8 \quad$ PICARDI, Nicola. "La vocazione del nostro tempo per la giurisdizione". Rivista trimestrale di diritto e procedura civile. Milano, marzo, 2004. pp. 41-42.

$9 \quad$ En este sentido, vale la pena ir a las observaciones precisas del jurista italiano Nicola Picardi: "In effetti oggi si avverte uno stato d'animo tendente a rivalutare il momento giurisprudenziale del diritto. È convizione difusa che il compito di adeguare il diritto alla realtà storico-social espetti anche al giudice e non è mancato chi, sai purê sotto diverse sfumature, abbia considerato la opera come fonte, concorrente e strumentale, di produzione giuridica. (...) Sorge, così, uma grave questione: fenomeni de genere possono segnare il passagio dal Gesetzstaat al Richterstaat, da uno Stato a prevalente potere legislativo ad uno Stato a prevalente potere giudiziario?" Op. cit. p. 42. 
Pietro Costa, al tejer sus concluyentes consideraciones en la obra referida, agrega: "Se em todo curso da sua história o Estado de Direito parece indicar no papel do juiz o enigma resolvido da juridicização do poder, resulta de qualquer modo, compreensível que a temática hodierna do Estado de Direito encontre propriamente na hermenêutica jurídica, nos problemas relativos ao papel do juiz, às técnicas de interpretação e aplicação do direito, o seu momento decisivo de verificação"10.

En este escenario, por lo tanto, se hace necesario en primer orden el estudio de la teoría de los precedentes vinculantes, ya que habita en el papel del juez para controlar la resolución del poder soberano, nada más lógico que la aplicación del derecho se produzca de manera uniforme y coherente, de acuerdo con el derecho a la igualdad que debe ser entendido tanto por el ángulo de las decisiones judiciales como por la ley ${ }^{11}$.

Neil MacCormick, al tratar el tema de la utilización de los precedentes en el Estado de Derecho, expone tres razones para justificar su adopción por parte de jueces y abogados. La primera es la razón de la justicia. En otras palabras, si el caso se refiere a hechos similares y los argumentos jurídicos propuestos son los mismos, no hay ninguna razón por la que el caso futuro sea juzgado de una manera contraria a los casos anteriores. La segunda razón se relaciona con la primera, una vez que tiene la intención de defender la imparcialidad del sistema legal que proporciona la misma justicia para todos, con independencia de las partes o del juez. La tercera trata de la economía de esfuerzo, es decir, no es necesario que los jueces y abogados realicen nuevos esfuerzos argumentativos para resolver una cuestión jurídica que ya se había decidido, a menos que el caso revele nuevos elementos de hecho que requieren mayor análisis y la reconsideración de los argumentos firmados en el precedente $^{12}$.

La transformación del Estado legislativo en el Estado constitucional liberal contemporáneo provocó un cambio en la concepción general de derecho, que se entrelaza con la propia evolución del sistema del civil law. La vieja teoría de que el sistema jurídico de los países adeptos al civil law tuvieron como perfil una jurisdicción que tenía la simple enunciación de la ley porque no había nada que interpretar o crear, ahora ya no existe, sobre todo en el ordenamiento jurídico del Brasil ${ }^{13}$.

$10 \quad$ Cf. COSTA, Pietro Op. cit. p. 196.

11 Sobre el principio de igualdad ante las decisiones judiciales, “Treat like cases alike”, cf. David Strauss. Must Like Cases Be Treated Alike? (May 2002). University of Chicago, Public Law Research Paper No. 24. Disponible en: http://ssrn.com/abstract=312180; MARMOR, Andrei. Should Like Cases be Treated Alike? (November 10, 2004). USC Law and Public Policy Research Paper No. 04-26. Disponible en: http://ssrn.com/abstract=618382.

12 MACCORMICK, Neil. Retórica e o Estado de Direito. Rio de Janeiro: Elsevier, 2008. p. 191.

13 Véase, en este punto, el estudio histórico-crítico llevado a cabo por el profesor Marinoni, que aborda el manejo del discurso actual sobre la adopción de precedente vinculante en el sis- 
Los factores determinantes de esta transformación ${ }^{14}$ habitan en el perfil del control de constitucionalidad de las leyes establecidas en Brasil, estas asignan a cada juez la facultad y el deber de determinar la constitucionalidad de la ley en el juicio del caso concreto, negando la validez de ésta por ser incompatible con la Constitución. No sólo eso, compete también al juez -en ausencia de regulaciones que impiden el ejercicio de los derechos constitucionales y las libertades y prerrogativas inherentes a la soberanía, la nacionalidad y la ciudadanía-, garantizar el derecho constitucional violado en el caso ${ }^{15}$.

Otro factor es la existencia de las llamadas cláusulas generales que exigen del juez ya no una actividad declarativa sino una actividad integradora, una vez que se vale de la cláusula general para llegar a la norma del caso particular, es decir, la creación de una verdadera norma jurídica del $\mathrm{caso}^{16}$, perfil de la Corte que se deriva del reconocimiento de la insuficiencia de la actividad legislativa en la sociedad política actual, dado el carácter incompleto de la norma jurídica que abarque la totalidad de los hechos sociales, así como la rápida mutación de las tecnologías y de los valores sociales.

Por lo tanto, en vista de este nuevo marco legal, marcado por el color de la jurisprudencia del caso, es necesario dar coherencia a el orden legal y a el ordenamiento jurídico producido por las Cortes Supremas (y aquí hablamos tanto de la jurisprudencia ordinaria como de la constitucional) e importar el valor de la seguridad jurídica y la igualdad, a través de la estabilidad de las decisiones judiciales con el fin de eliminar el conflicto con los valores del Estado constitucional. Y lo más importante, garantizar la materialización del Estado de Derecho, a través

tema de derecho civil, en particular en el ordenamiento jurídico brasileño. Cf. Precedentes Obrigatórios. São Paulo, Ed. RT, 2010. pp. 58-88.

14 Ver, sobre los factores determinantes, PICARDI, Nicola. Op. cit. p. 43, sobre la influencia del control de constitucionalidad, en particular, MARINONI, Luiz Guilherme. Op. cit. pp. 67-85.

15 Aquí la acción se centra en el amparo constitucional, de conformidad con el art. 5, párrafo LXXI, la CRFB: orden de Subvenciones, donde la falta de regulación hace que sea imposible el ejercicio de los derechos y libertades constitucionales y las prerrogativas inherentes a la nacionalidad, la soberanía y la ciudadanía. Ver, en este punto, la evolución de la jurisprudencia de la Corte Suprema de Justicia relativa a la actuación de la jurisdicción constitucional, como un ejemplo, 721/DF MI, rel. Min. Marco Aurelio DJE publicado en 30/11/2007, en la que se declaró que estaba en manos de los tribunales con arreglo al art. 5, párrafo LXXI. La CRFB no sólo emitirá un certificado de omisión del poder encomendado para regular el derecho de las libertades constitucionales, las prerrogativas inherentes a la nacionalidad, la soberanía y la ciudadanía, pero factible en este caso, el ejercicio de ese derecho a la consecuencias de la inacción por parte de la legislatura.

16 Ver MARINONI, Luiz Guilherme. Teoria Geral do Processo. 5a Edição. São Paulo: Revista dos Tribunais, 2011. pp. 121-124; TARUFFO, Michele. "Legalità e giustificazione della creazione giudiziaria del diritto". Rivista trimestrale di diritto e procedura civile. Milano, marzo-2001. pp. 15-19. 
del freno del poder judicial que no puede actuar libremente en la aplicación, con el fin de provocar una situación de desventaja para los ciudadanos.

La búsqueda de la realización de un sistema legal compatible con el establecimiento de un Estado Democrático de Derecho, tiene como valor moral la previsibilidad y como valores el determinar la estabilidad de las decisiones judiciales, la seguridad jurídica y la igualdad de estos jurisdicionados.

Es cierto que un sistema jurídico caracterizado por la dispersión y la imprevisibilidad de las decisiones judiciales es un serio obstáculo para el desarrollo de las actividades económicas del país, así como causa de su frontal potencial deslegitimador frente los ciudadanos, en la medida en que el propio Poder Judicial no respeta sus decisiones. Ahora, todo ciudadano tiene derecho a conocer, con precisión aguda, si un acto es ilegal o no y cuáles son las consecuencias jurídicas de la práctica de su conducta ${ }^{17}$.

Aunque se defienda en este ensayo la previsibilidad como un valor moral, que corresponde con el valor de proteger las expectativas justificadas, esta confianza justificada del jurisdicionado debe ser, sin duda, razonable y legítima, de acuerdo con la práctica legal en su Estado. Es decir, sería correcto decir ¿qué litigantes potenciales que dependían de las decisiones judiciales anteriores y formaban expectativas sobre la ley, en un sistema en el que las decisiones judiciales no tienen fuerza vinculante pero persuasiva, tuvieron sus expectativas frustradas, y por lo tanto derecho a la igualdad de trato? ¿Cómo podemos definir el momento en que hubo una justificada expectativa del litigante? En esta línea de argumentación es oportuna la prudencia propuesta por Andrei Marmor. Este autor dice que "parte de lo que hace las expectativas sobre la ley razonable y legítima es que el corresponde al tipo de expectativas apoyada por la práctica jurídica. Para dar un ejemplo extremo, en un sistema jurídico que no reconoce la doctrina del precedente los litigantes potenciales no pueden depender de las decisiones judiciales para hacer predicciones sobre la ley legítima. Si la ley dice que las decisiones de los tribunales superiores no son vinculantes, no se puede tener una expectativa legítima de qué serían. Por lo tanto, la cuestión de cuáles son las expectativas legítimas sobre la ley es, en parte, pero fundamentalmente, una cuestión de práctica judicial”18.

17 En este sentido, John Dewey, considerado uno de los fundadores del realismo jurídico americano: "Another moving force is the undoubted need for the maximum possible of stability and regularity of expectation in determining courses of conduct. Men need to know the legal consequences which society through the courts will attach to their specific transactions, the liabilities they are assuming, the fruits they may count upon in entering upon a given course of action. This is a legitimate requirement from the standpoint of the interests of the community and of particular individuals." Op. cit. pp. 24-25.

18 En sus propias palabras: "Perhaps a much stronger case for the principle of treating like cases a like can be made by reference to the value of protected expectations. Potential litigants may have relied on previous judicial rulings and formed expectations about the law that it would be wrong to frustrate. But we must proceed with caution here: Not every expectation potential 
Teniendo en cuenta esta precaución, entre otras razones establecidas en el presente trabajo, se defiende aquí la institucionalización de una doctrina del precedente vinculante. Por otro lado, la ausencia de legislación específica, sea constitucional $\mathrm{u}$ ordinaria sobre lo referente a los precedentes judiciales, no puede tomarse como justificación para su fracaso, la derrota debe ser vista como verdadera falta de seguridad jurídica, el verdadero fracaso del juez. El respeto a los precedentes no depende de una norma legal que indica su requerimiento expreso, aquí la adopción deriva de la existencia del Estado de Derecho que tiene por objeto la protección de la seguridad jurídica y de la certeza del derecho, y con estos el desarrollo del país.

En este sentido, la adopción de un precedente vinculante es mucho más esencial en el ordenamiento jurídico brasileño debido a que la Constitución atribuye a los Tribunales Superiores, órganos centrales dentro del campo de la división diseñada por la Constitución Federal, la función primordial de garantizar la unidad de la interpretación de la ley, ya sea constitucional o federal, así como la protección de la igualdad sustancial de los derechos fundamentales de los ciudadanos, connotando así el instrumento necesario para garantizar la consistencia y la coherencia de un sistema jurídico complejo como el nuestro ${ }^{19}$.

Con respecto a los aspectos finales, la adopción de lo anterior se justifica en la medida en que trata de fomentar unos valores que están relacionados, a saber, con la seguridad jurídica, la previsibilidad, la uniformidad de la ley, la igualdad entre los ciudadanos, la credibilidad de los tribunales, la reducción de los litigios y la preservación de la fuerza normativa de la Constitución, en virtud del respeto a la exégesis de autoridad producida por el Supremo Tribunal.

Por lo tanto, no tiene nada que ver con el cercenamiento de la libertad de juzgar que tiene el juez, según ha señalado la doctrina disidente del precedente vinculante. Ésta sigue siendo inquebrantable, porque no se desea el final de la libre convicción judicial, tanto es así que las "verdades procesuales", creadas por la evidencia de que contribuyan con el registro o la obligación de las partes, puede causar diferentes soluciones jurídicas en relación con las controversias que, vistas desde a cabo por los ciudadanos, parecen ser las mismas. Es decir, los hechos

litigants may have about the law is one which it would be wrong to frustrate. The expectation must be reasonable and legitimate. Part of what makes expectations about the law reasonable and legitimate is that the relevant type of expectations is supported by legal practice. To take an extreme example, in a legal system that does not recognize a doctrine of precedent, potential litigants cannot relyon judicial decisions to make legitimate predictions about the law. If the law tells you that the decisions of higher courts are not binding, you cannot have a legitimate expectation that they would be. So the question of what expectations about the law are legitimate is partly, but crucially, a matter of legal practice." Op. cit. p. 9.

19 Ver DA SILVA, Ovídio A. Batista. "A função dos Tribunais Superiores". En: Sentença e coisa julgada (ensaios e pareceres). 4a. ed. Rio de Janeiro: Forense, 2003. 
pueden conducir a resultados diferentes, sin embargo, la aplicación de la ley debe ser igual para todos ${ }^{20}$.

Vale la pena señalar que, cuando se trata aquí de un precedente vinculante, se habla de la adopción de la norma jurídica coherente que debe ser observada por los tribunales en casos similares, a fin de evitar la lotería judicial, donde las exigencias legales similares reciben soluciones de manera diferente. Sin embargo, no sostenemos aquí que la uniformidad de las decisiones, que son dictadas por el principio de igualdad ante las decisiones judiciales (que se tratan como casos iguales), supone la eliminación de la capacidad de los tribunales para tomar decisiones diferentes en circunstancias similares, cuando esto tiene que reflejar las preferencias de diferentes, debido a la diversidad y el pluralismo ${ }^{21}$. Por supuesto, el principio de uniformidad de las decisiones en algunos casos no es deseable, debido a las particularidades del caso no lo aceptan, lo que requiere una nueva decisión que tome en cuenta las diferencias. Y esta diferenciación, al contrario de lo que podría suponerse, es deseable. Sin embargo, para ello, corresponde al órgano jurisdiccional el utilizar un mayor esfuerzo argumentativo para demostrar la peculiaridad de este caso y la eliminación de la regla anterior. Lo que no es posible ni aceptable es la marginación de los precedentes, cuando el caso presenta los mismos hechos y los mismos argumentos jurídicos.

Por tanto, es de vital importancia conocer la forma de aplicar el precedente, que lo identifica como algo que, al tiempo que guía a la gente y obriga los jueces, no inmobiliza las relaciones sociales o impide la jurisdicción de producir un derecho en consonancia con la realidad y con los nuevos tiempos ${ }^{22}$. Debe darse cuenta de

20 HERNANDO, Francisco José. "Jurisprudencia vinculante:uma necesidade del Estado de Derecho". Revista de Economía y Derecho, Vol. 3, n 10, 2006. p 78.

21 Análisis hecho, en razón de la objeción propuesta por Andrei Marmor: "Thus, allowing the courts to make different choices under similar circumstances may be justified by the need to cater to different populations with divergent needs and preferences. As long as the relevant judicial decisions are morally and otherwise within the bounds of permissibility (as we have assumed all along), courts should be allowed, at least in certain cases, to reach different decisions that reflect different preferences amongst otherwise incommensurable values. The uniformity of decisions that is dictated by the principle of treating like cases alike seriously undermines the courts' ability to respond to the needs of diversity and pluralism. To be sure, I am not suggesting that these reasons for forgoing uniformity are necessarily present in each and every case. Far from it. But often one, or both of them, are, and this should count against the assumption that the value of predictability of judicial decisions ought to prevail. Thus, treating like cases alike is not necessarily desirable or even warranted." Op. cit. p 13.

22 En este sentido, Andrei Marmor argumenta: "Increasing the predictability of judicial decisions is never costless, and often the costs will outweigh the benefits. The more judicial decisions are predictable, the more rigid they are. Generally speaking, rigidity is undesirable because it tends to impede judicial experimentation, innovation, and other, potentially desirable, social changes. It is a truism, but none the less an important one, that there is always a delicate bal- 
que la adopción del sistema de precedente vinculante no implica la inmovilización del derecho ni la restricción de la libertad de juzgar al juez ${ }^{23}$.

Es aquí necesario señalar que, aunque cualquier precedente tenga un contenido, esto puede ser limitado o ampliado en función de las necesidades de los nuevos casos, permitiendo así el desarrollo de lo expresado arriba y por lo tanto del derecho.

Además, como ningún precedente pretende ser para siempre válido, hay que tener en cuenta los criterios que, sin violar la confianza justificada, permiten su derogación. Teniendo en cuenta que el precedente obligatorio tiene como objeto formar el derecho y proteger la seguridad jurídica, es por igual esencial para hacer frente a la posibilidad de anticipar la derogación del precedente y de la modulación de los efectos de la decisión que lo dejan de lado, a fin de evitar la aplicación injusta de los precedentes gastados y, en la segunda hipótesis, proteger a los que creen en la decision judicial ${ }^{24}$.

\section{ConCLusión}

El Estado de Derecho, como la piedra angular del Estado constitucional, tiene viejos argumentos, que pueden ser definidos en la relación entre poder y derecho. La lucha por el Estado de Derecho implica la limitación de la actividad estatal al derecho como tal, resultado del cumplimiento del objetivo del sistema, con el fin de favorecer una situación más ventajosa para el individuo.

La sociedad es anterior al Estado y aplaza una parte de su libertad a este para garantizar un nivel mínimo de civilidad social entre los individuos, a partir de la aplicación de las reglas firmadas en el contrato social. En esta medida, los ciudadanos demandan un ambiente cívico, en que puedan vivir con sus derechos respetados y certos de la protección de cierta seguridad en sus relaciones.

Sin embargo, la transformación del papel de la ley, la pérdida de su "Ilustración aura" y su uso cada vez más como un instrumento de la acción del gobierno y no como una representación de la voluntad general del pueblo, el papel del juez y su relación con el control de observancia de las leyes y normativas de un parámetro, repropondrán el debate sobre la expresión Estado de Derecho.

El Estado de Derecho surge, en nuestro tiempo histórico, con una vocación definida, la jurisdicción, el control del Estado por parte de la Corte Constitucional

ance that needs to be maintained between the values of predictability of judicial decisions and the values which call for their flexibility". Op. cit. p. 11.

${ }^{23}$ Como afirma Benjamim Cardozo: "Meu dever de juiz talvez seja materializar em lei não as minhas aspirações, convicções e filosofia pessoais, mas as aspirações dos homens e mulheres do meu tempo." CARDOZO, Benjamin N. A natureza do processo judicial. São Paulo: Martins Fontes, 2004. p. 128.

24 Ver: Marinoni, Luiz Guilherme. Precedentes obrigatórios. Op. cit. pp. 388-425. 
que tiene el poder de controlar la constitucionalidad de las leyes, declarando si es válida o no para un caso ordinario o incluso para rechazar el sistema legal basado la fiscalización abstracta de la ley.

Esta vocación por la jurisdicción, sin embargo, conlleva algunos problemas que ponen en juicio el propio Estado de Derecho, ya que permite la creación de un ambiente de inseguridad jurídica debido a la falta de previsibilidad de la definición de la interpretación de la ley. Problema que crece con el control difuso sin la implementación del stare decisis, pues, al competerle a cualquier juez decidir sobre la constitucionalidad de una ley específica, decisiones varias, incluso contrarias, van a coexistir en el ordenamiento jurídico, lo que a su vez provoca la fragilidad del Estado de Derecho.

Con el objetivo de solucionar este problema es que se propone adoptar, en el ordenamiento jurídico brasileño, la teoría de los precedentes vinculantes, como medio de asegurar la protección de la seguridad jurídica, la previsibilidad y la certeza.

Es cierto que la resolución de los precedentes judiciales vinculantes no agota los problemas de la relación entre el poder y la ley. Sin embargo, permitirá el cerramiento del sistema jurídico en lo que respecta a controlar las acciones del poder judicial, que deben ser guiadas por la coherencia, la igualdad de las decisiones en casos similares y la seguridad jurídica.

Después de todo, queda claro la posibilidad de conexión temática entre el Estado de Derecho y la protección de la seguridad jurídica, como una forma de posición favorable dado a los sujetos en el imperio de la ley.

\section{REFERENCIAS}

BAPTISTA DA SILVA, Ovídio A. "A função dos Tribunais Superiores". In: Sentença e coisa julgada (ensaios e pareceres). 4ª . ed. Rio de Janeiro : Forense, 2003.

CARDOZO, Benjamin N. A natureza do processo judicial. São Paulo: Martins Fontes, 2004.

COSTA, Pietro. "O estado de direito: uma introdução histórica". In: COSTA, Pietro; ZOLO, Danilo (Org.) O estado de direito, história, teoria, crítica. São Paulo: Martins Fontes, 2006.

DEWEY, John. Logical Method and Law. 10 Cornel Law Quartely 17, 1914/1925.

ENTERRÍA, Eduardo Garcia de. La constitución como norma y el tribunal constitucional. Madrid: Editorial Civitas, S.A, 1985.

HERNANDO, Francisco José. "Jurisprudencia vinculante: una necesidade del Estado de Derecho". Revista de Economía y Derecho, Vol. 3, n 10, 2006.

MACCORMICK, Neil. Retórica e o Estado de Direito. Tradução Conrado Hübner Mendes. Rio de Janeiro: Elsevier, 2008. 
MARINONI, Luiz Guilherme. Precedentes obrigatórios. São Paulo: Revista dos Tribunais, 2010. Tribunais, 2011. Teoria Geral do Processo. $5^{a}$ Edição. São Paulo: Revista dos

MARMOR, Andrei. "Should Like Cases be Treated Alike?" (November 10, 2004). USC Law and Public Policy Research Paper No. 04-26. Disponible en: http:// ssrn.com/abstract $=618382$. . "The Rule of Law and Its Limits" (April 2003). USC Law and Public Policy Research Paper No. 03-16. Disponível em: http://ssrn.com/abstract=424613.

PICARDI, Nicola. La vocazione del nostro tempo per la giurisdizione. Rivista trimestrale di diritto e procedura civile. Milano, marzo, 2004.

STRAUSS, David A. "Must Like Cases Be Treated Alike?" (May 2002). U of Chicago, Public Law Research Paper No. 24. Disponible en: http://ssrn.com/abstract $=312180$.

ROSENFELD, Michel. "The rule of law and the legitimacy of constitutional democracy". Southern California Law Review, vol. 74, 2001.

TARUFFO, Michele. "Legalità e giustificazione della creazione giudiziaria del diritto". Rivista trimestrale di diritto e procedura civile. Milano, marzo, 2001.

VERDÚ, Pablo Lucas. A luta pelo Estado de Direito. Rio de Janeiro: Editora Forense, 2007. 